\title{
Dynamics of a seismogenic fault subject to variable strain rate
}

\author{
M. Dragoni ${ }^{1}$ and A. Piombo ${ }^{2}$ \\ ${ }^{1}$ Dipartimento di Fisica, Alma Mater Studiorum - Università di Bologna - Viale Carlo Berti Pichat 8, 40127 Bologna, Italy \\ ${ }^{2}$ Dipartimento di Fisica and C.I.R.S.A., Alma Mater Studiorum - Università di Bologna - Viale C. Berti Pichat 8, \\ 40127 Bologna, Italy
}

Received: 21 February 2011 - Revised: 20 June 2011 - Accepted: 22 June 2011 - Published: 29 June 2011

\begin{abstract}
The behaviour of seismogenic faults is generally investigated under the assumption that they are subject to a constant strain rate. We consider the effect of a slowly variable strain rate on the recurrence times of earthquakes generated by a single fault. To this aim a spring-block system is employed as a low-order analog of the fault. Two cases are considered: a sinusoidal oscillation in the driver velocity and a monotonic change from one velocity value to another. In the first case, a study of the orbit of the system in the state space suggests that the seismic activity of the equivalent fault is organized into cycles that include several earthquakes and repeat periodically. Within each cycle the recurrence times oscillate about an average value equal to the recurrence period for constant strain rate. In the second case, the recurrence time changes gradually from the value before the transition to the value following it. Asymptotic solutions are also given, approximating the case when the amplitude of the oscillation or of the monotonic change is much smaller than the average driver velocity and the period of oscillation or the duration of the transition is much longer than the recurrence times of block motions. If the system is not isolated but is subject to perturbations in stress, the perturbation anticipates or delays the subsequent earthquake. The effects of stress perturbations in the two cases of strain rate oscillations and monotonic change are considered.
\end{abstract}

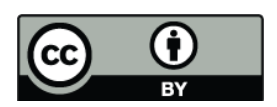

Correspondence to: A. Piombo (antonello.piombo@unibo.it)

\section{Introduction}

If we consider the simplest stick-slip model for the earthquake mechanism, such as the one proposed one century ago by Reid (1911), fault slip events are found to be periodic and predictable. However observations reported in seismic catalogues show that seismic fault slips occur aperiodically. Many attempts have been made to ascertain the reasons of aperiodicity and hence to achieve a deeper knowledge of the earthquake mechanism.

In particular, attempts to reproduce the long-term correlations of earthquakes have been made by adding Brownian perturbations to steady tectonic loading (Matthews et al., 2002; Zöller and Hainzl, 2007), by studying the stress evolution in discrete fault models (Ben-Zion et al., 2003; Zöller et al., 2007) and by using the concept of self-organized criticality (Abaimov at al., 2007; Baiesi, 2009). Fault interaction is predicted on theoretical grounds (e.g. Steacy et al., 2005) and is found to be relevant to the long-term behaviour of fault systems (Marzocchi et al., 2003).

When investigating the long-term behaviour of a fault system, models with a finite number of degrees of freedom are often preferable to descriptions based on continuum mechanics, since they allow long-term properties to be studied in a finite-dimensional state space. Such low-order analogs of seismic sources are spring-block systems that were first proposed by Burridge and Knopoff (1967). Following authors have shown that such systems can simulate several features of seismic activity (Dieterich, 1972; Rundle and Jackson, 1977; Cohen, 1977; Cao and Aki, 1984, 1986; Gu et al., 1984; Carlson and Langer, 1989a, b; Huang and Turcotte, 1990b; Carlson et al., 1994).

Published by Copernicus Publications on behalf of the European Geosciences Union and the American Geophysical Union. 
A single block pulled by a spring on a rough surface reproduces the simple Reid's elastic rebound mechanism. Following models have mainly explored two possibilities. One is the role of the constitutive equation of the fault. The simplest friction law that generates a stick-slip behaviour is a piecewise constant function of slip rate, with friction assuming a static or a dynamic value. Many models have assumed more complicated friction laws which are obtained from laboratory experiments (Byerlee, 1978; Ruina, 1983; Rice and Tse, 1986; Gu and Wong, 1991; de Sousa Vieira, 1995; Belardinelli and Belardinelli, 1996; Erickson et al., 2008).

The other process which has been considered is fault interaction. This has been done with systems made of two coupled blocks, representing two fault asperities or fault segments. Nussbaum and Ruina (1987) and Turcotte (1997) considered a two-block model with spatial symmetry and found that the system can exhibit periodic orbits in the state space, representing the alternate motion of blocks. Huang and Turcotte (1990a, 1992), McCloskey and Bean (1992) and He (2003) showed that a two-block model without spatial symmetry may yield chaotic behaviour. Dragoni and Santini (2010) suggested that aperiodicity may be the effect of stress transfers from neighbouring faults preventing the system from settling down in a periodic orbit.

In the present paper, a third possibility is investigated. We assume that the tectonic strain rate which is imposed to a seismogenic fault is slowly variable in time. There is evidence that the velocities of tectonic plates are not constant in time, but undergo changes in the very long term (e.g. King et al., 2002; Iaffaldano and Bunge, 2009). These may be due to changes in the velocity of mantle convection. We consider two cases: a sinusoidal oscillation in velocity and a monotonic change from one velocity value to another. Typically the oscillations or the transitions in plate velocity will have smaller amplitudes and longer periods and durations than recurrence times of earthquakes. This is modelled by a springblock system where the driver velocity is a slowly varying function of time. We consider the simplest friction law, characterized by a static and a dynamic friction. The system is dissipative and nonlinear. As a premise we briefly review the dynamics of a spring-block system with constant driver velocity; then we study the evolution of the system with variable velocity.

\section{Constant driver velocity}

We consider a heavy block with mass $m$ placed on a horizontal plane. A horizontal spring with rigidity $K$ connects the block to a driver moving at constant velocity $v$ away from the block and along the spring axis. We assume that the motion of the block is resisted by a static friction $f_{\mathrm{s}}$ and a dynamic friction $f_{\mathrm{d}}$, and define $\epsilon=\frac{f_{\mathrm{d}}}{f_{\mathrm{s}}}$

where $0<\epsilon<1$. The state of the system can be represented by the extension $x$ of the spring as a function of time $t$ and the force exerted by the spring on the block is

$f=-K x$

Following Turcotte (1997), we introduce the nondimensional quantities

$X=\frac{K x}{f_{\mathrm{s}}}, \quad T=\sqrt{\frac{K}{m}} t, \quad V=\frac{\sqrt{K m}}{f_{\mathrm{s}}} v$.

The dynamical system is defined by the equations

$\dot{X}=Y, \quad \dot{Y}=0$

when the block is stationary, and

$\dot{X}=Y, \quad \dot{Y}=\epsilon-X$

when the block is moving, where dots indicate differentiation with respect to $T$. In writing Eq. (5), we have assumed that $v$ is much smaller than the block velocity, since the ratio between the velocity of tectonic plates and the slip rate of a fault is in the order of $10^{-9}$. This means that the driver is virtually stationary during the motion of the block. With initial conditions

$X(0)=X_{0}, \quad Y(0)=V$

the solution of Eq. (4) is

$X(T)=X_{0}+V T, \quad Y(T)=V$.

If we define a nondimensional force

$F=\frac{f}{f_{\mathrm{s}}}$

the block starts moving when $F=-1$ or $X=1$. With initial conditions

$X(0)=1, \quad Y(0)=0$

the solution of Eq. (5) is

$X(T)=\epsilon+\frac{U}{2} \cos T, \quad Y(T)=-\frac{U}{2} \sin T$

where $0 \leq T \leq \pi$ and

$U=2(1-\epsilon)$

is the displacement of the block. Since changes in the sign of shear traction are not observed on faults after an earthquake, we only consider the case $F \leq 0$ or $X \geq 0$, implying $\epsilon \geq 1 / 2$. When the block motion stops, the sliding condition is reached again after a time 


$$
\Delta T=\frac{U}{V}
$$

which is the recurrence period of block motions.

The state space of the system is a subset of $R^{2}$. As discussed above, $V$ is extremely small with respect to the values that $Y$ assumes when the block is moving. In drawing the orbits of the system, we may therefore assume $Y=0$ in Eq. (7). Since a fault is stationary for most of its lifetime, it is natural to assume a point $P_{0}=\left(X_{0}, 0\right)$ as initial point.

According to Eqs. (7) and (10), the orbit is a segment of the $X$ axis up to $P_{1}=(1,0)$, then it describes a half circumference in the half-plane $Y<0$, with centre at point $(\epsilon, 0)$ and radius $U / 2$. The orbit intersects the $X$ axis at $P_{2}=(1-U, 0)$, then continues along the $X$ axis to $P_{1}$, and so on. The system is then characterized by limit cycles which are the union of a segment and a half-circumference (Fig. 1), with a radius depending on $\epsilon$ and a period equal to $\Delta T$. Variations of $\epsilon, f_{\mathrm{s}}$ or $v$ with time may render aperiodic the motion: we investigate the effect of varying $v$.

\section{Variable driver velocity: sinusoidal oscillation}

We assume that the driver velocity oscillates about the value $v$ with amplitude $a$ and frequency $\omega$ and introduce the nondimensional quantities

$A=\frac{a}{v}, \quad \Omega=\sqrt{\frac{m}{K}} \omega$.

Therefore we write

$Y(T)=V(1+A \sin \Omega T)$

where we have assumed that velocity is equal to $V$ at $T=0$. The ratio between $\Omega$ and the frequency of block motions in the case of constant velocity is

$\alpha=\frac{\Omega \Delta T}{2 \pi}$.

It is appropriate to introduce a coordinate

$$
Z=\Omega T
$$

which is regarded as an angle varying from 0 to $2 \pi$ : therefore $Z$ will be intended as modulo $2 \pi$. When the block is stationary, the dynamical system is described by the system of autonomous differential equations

$\dot{X}=Y, \quad \dot{Y}=A V \Omega \cos Z, \quad \dot{Z}=\Omega$.

With initial conditions

$X\left(T_{0}\right)=X_{0}, \quad Y\left(T_{0}\right)=Y_{0}, \quad Z\left(T_{0}\right)=Z_{0}$

the solution is

$X(T)=X_{0}+V\left(T-T_{0}\right)-\frac{A V}{\Omega}\left(\cos \Omega T-\cos \Omega T_{0}\right)$

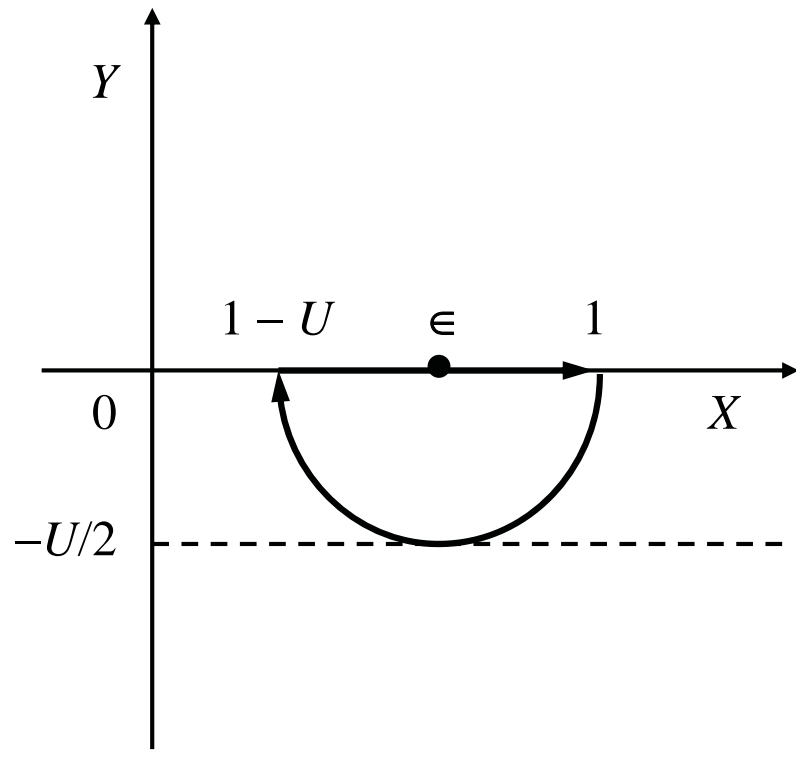

Fig. 1. A limit cycle in the state space of the spring-block system with constant driver velocity.

$Y(T)=Y_{0}+A V \sin \Omega\left(T-T_{0}\right)$

$Z(T)=Z_{0}+\Omega\left(T-T_{0}\right)$

which are the parametric equations of the orbit. If $A \ll 1$, Eq. (19) describes a curve that slightly oscillates about the line

$X(T)=X_{0}+V\left(T-T_{0}\right)$

According to Eqs. (19) and (20), the projection of the orbit in the plane $X Y$ is a curtate cycloid enclosed between the lines $Y=(1-A) V$ and $Y=(1+A) V$. When the block is moving, the system of differential equations is given by Eq. (5) with the addition of the equation for $\dot{Z}$ in (17). The solution is given by Eq. (10) with the addition of Eq. (21). Hence the orbit is the union of cycloid segments and half circumferences winding in a 3-manifold homeomorphic to a solid torus. As initial conditions we choose

$T_{0}=0, \quad X_{0}=1-U, \quad Y_{0}=V, \quad Z_{0}=0$.

Since the driver velocity is much smaller than the block velocity, in drawing the orbits we can approximate the cycloids with straight lines lying in the plane $Y=0$ and neglect the duration of block motions with respect to the duration of periods in which the blocks are stationary (Fig. 2). Then the state space is restricted to

$1-U \leq X \leq 1, \quad-U / 2 \leq Y \leq 0, \quad 0 \leq Z \leq 2 \pi$

where $0<U \leq 1$ 


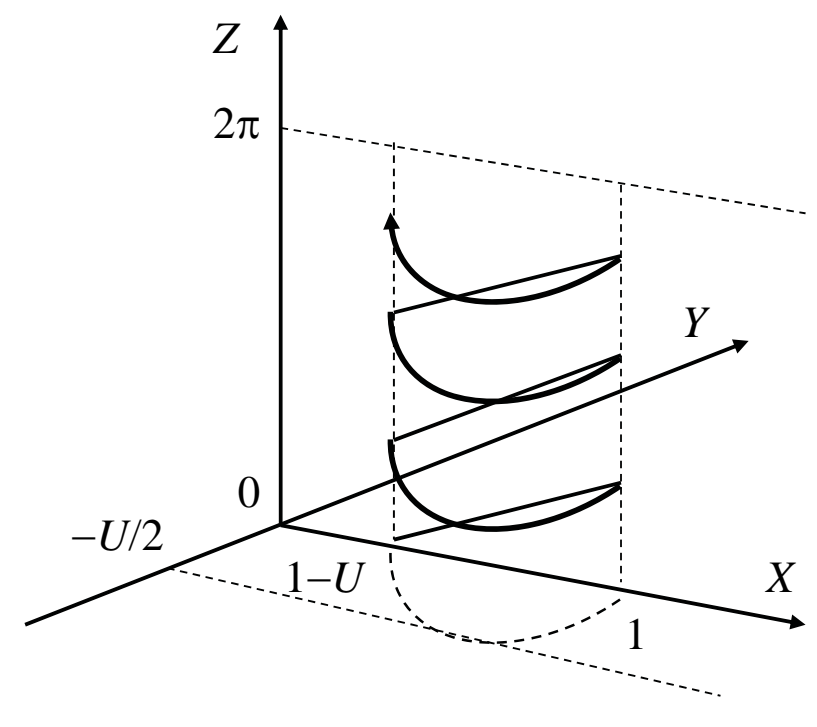

Fig. 2. Sketch of an orbit in the state space of the spring-block system with variable driver velocity, under the assumptions $A \approx 0$ and $V \approx 0$.

\section{Recurrence times}

Let us call $T_{i}$ the instant of time when the $i$-th block motion takes place and define

$Z_{i}=\Omega T_{i}, \quad i=1,2,3, \ldots$

The time intervals between two consecutive block motions (recurrence times) are then

$\Delta T_{i}=T_{i}-T_{i-1}$.

If we set $T_{0}=T_{i-1}$ and $X_{0}=1-U$, Eq. (19) yields

$$
\begin{aligned}
X(Z)= & 1-U+\frac{U}{2 \pi \alpha}\left(Z-Z_{i-1}\right) \\
& -\frac{A U}{2 \pi \alpha}\left(\cos Z-\cos Z_{i-1}\right)
\end{aligned}
$$

where we have used Eqs. (15), (16) and (25). The value of $Z_{i}$ can be calculated from the condition for block motion

$X\left(Z_{i}\right)=1$

which gives

$Z_{i}-Z_{i-1}-2 \pi \alpha-A\left(\cos Z_{i}-\cos Z_{i-1}\right)=0$.

Summing over $i$ from 1 to $k$ and considering that $Z_{0}=0$, we obtain
$Z_{k}-2 \pi \alpha k-A\left(\cos Z_{k}-1\right)=0$

that can be solved numerically for $Z_{k}$. The $i$-th recurrence time is then

$\Delta T_{i}=\frac{Z_{i}-Z_{i-1}}{2 \pi \alpha} \Delta T$.

With initial conditions (23), the projection of the orbit in the plane $X Z$ is a saw-tooth curve enclosed between the lines $X=1-U$ and $X=1$. The teeth are different from each other, having the same height $U$, but different widths. The $k$-th tooth is

$X_{k}(Z)=1+U \frac{Z-Z_{k}}{Z_{k}-Z_{k-1}}, \quad Z_{k-1} \leq Z \leq Z_{k}$.

Since $X=-F$ and $Z$ is proportional to time, Eq. (32) resembles a typical stress-time curve for slip-predictable models of earthquake occurrence. The interval $0 \leq Z \leq 2 \pi$ corresponds to one cycle of the driver velocity $Y$. Points $Z=Z_{k}$ correspond to block motions and their number depends on the value of $\alpha$. From Eq. (26), the associated time is

$T_{k}=\sum_{i=1}^{k} \Delta T_{i}$

Since the set of rational numbers is dense in $R$, we may assume that $\alpha$ is rational, i.e.

$\alpha=\frac{m}{n}$

where $m$ and $n$ are positive integers, with $m \ll n$. From Eqs. (15) and (34)

$\frac{2 m \pi}{\Omega}=n \Delta T$.

Hence $m$ cycles of $Y$ correspond to $n$ average recurrence times. The $m$-th cycle of $Y$ terminates with the $n$-th block motion, so that

$Z_{n}=2 m \pi$

and the initial conditions are recovered. According to Eqs. (25), (35) and (36), this occurs at time

$T_{n}=\frac{2 m \pi}{\Omega}=n \Delta T$.

The system is in a periodic orbit representing a sequence of $n$ block motions, with a period which is a multiple of the period $2 \pi / \Omega$ of the driver velocity.

\section{Variable driver velocity: monotonic change}

We now suppose that the driver velocity has a monotonic change from $V_{a}$ to $V_{b}$ in a finite time $\Theta$. Assuming that the change begins at $T=0$, we write

$Y(T)= \begin{cases}V_{a}, & T<0 \\ V_{+}\left(1+B \cos \frac{\pi T}{\Theta}\right), & 0 \leq T \leq \Theta \\ V_{b}, & T>\Theta\end{cases}$ 
where

$V_{+}=\frac{V_{a}+V_{b}}{2}, \quad V_{-}=\frac{V_{a}-V_{b}}{2}, \quad B=\frac{V_{-}}{V_{+}}$.

The driver velocity increases if $V_{b}>V_{a}$; it decreases if $V_{b}<V_{a}$. When the velocity is constant and equal to $V_{a}$ or $V_{b}$, the recurrence times are respectively

$\Delta T_{a}=\frac{U}{V_{a}}, \quad \Delta T_{b}=\frac{U}{V_{b}}$.

We also define

$\overline{\Delta T}=\frac{U}{V_{+}}, \quad \beta=\frac{\overline{\Delta T}}{2 \Theta}$

and introduce a coordinate

$Z=\frac{\pi T}{\Theta}$

which is regarded as an angle varying from 0 to $\pi$. When the block is stationary, the dynamical system is described by the system of equations

$\dot{X}=Y, \quad \dot{Y}=-\frac{\pi}{\Theta} V_{-} \sin Z, \quad \dot{Z}=\frac{\pi}{\Theta}$.

With initial conditions

$X(0)=1-U, \quad Y(0)=V_{a}, \quad Z(0)=0$

the solution is

$X(T)=1-U+V_{+}\left(T-T_{0}\right)+V_{-} \frac{\Theta}{\pi}\left(\sin \frac{\pi T}{\Theta}-\sin \frac{\pi T_{0}}{\Theta}\right)$

$Y(T)=V_{+}+V_{-} \cos \frac{\pi T}{\Theta}, \quad Z(T)=\frac{\pi T}{\Theta}$.

Similar considerations to those expressed for the sinusoidal velocity oscillation hold, with the difference that the orbit is enclosed between the lines $Y=V_{a}$ and $Y=V_{b}$ in the plane $X Y$ and $Z$ varies only between 0 and $\pi$.

We call $T_{i}$ the instant of time when the $i$-th block motion takes place in the interval $0<T<\Theta$ and define

$Z_{i}=\frac{\pi}{\Theta} T_{i}, \quad i=1,2,3, \ldots$

The recurrence times are given by Eq. (26). If we set $T_{0}=T_{i-1}$, Eq. (45) yields

$$
\begin{aligned}
X(Z)= & 1-U+\frac{U}{2 \pi \beta}\left(Z-Z_{i-1}\right) \\
& +\frac{B U}{2 \pi \beta}\left(\sin Z-\sin Z_{i-1}\right) .
\end{aligned}
$$

The value of $Z_{i}$ is calculated from condition (28), which gives

$Z_{i}-Z_{i-1}-2 \pi \beta+B\left(\sin Z_{i}-\sin Z_{i-1}\right)=0$.
Summing over $i$ from 1 to $k$ and considering that $Z_{0}=0$, we obtain

$Z_{k}-2 \pi \beta k+B \sin Z_{k}=0$

that can be solved numerically for $Z_{k}$. The $i$-th recurrence time is then

$\Delta T_{i}=\frac{Z_{i}-Z_{i-1}}{2 \pi \beta} \overline{\Delta T}$.

A saw-tooth curve is obtained again in the plane $X Z$. Points $Z=Z_{k}$ correspond to block motions and their number in the interval $0<Z \leq \pi$ depends on the value of $\beta$. The associated time is given by Eq. (33).

We assume that $n$ earthquakes $(n \geq 1)$ take place in the time interval $0<T \leq \Theta$. In the particular case

$\beta=\frac{1}{2 n}$

we have $\Theta=n \overline{\Delta T}$ so that $T_{n}=\Theta$ and $\Delta T_{n+1}=\Delta T_{b}$. Otherwise $T_{n}<\Theta$ and the occurrence time $T_{n+1}$ is obtained from the condition

$X\left(T_{n+1}\right)=1$

whence

$T_{n+1}=\Theta+\frac{1-X(\Theta)}{V_{b}}$

where, from Eq. (45) with $T_{0}=T_{n}$,

$X(\Theta)=1-U+V_{+}\left(\Theta-T_{n}\right)-V_{-} \frac{\Theta}{\pi} \sin \frac{\pi T_{n}}{\Theta}$.

Then

$\Delta T_{n+1}=T_{n+1}-T_{n}$

Afterwords

$\Delta T_{k}=\Delta T_{b}, \quad k>n+1$

\section{Asymptotic solutions}

The motion of tectonic plates is observed to be very stable in the short term. In the case of sinusoidal oscillations, we may therefore assume

$A \ll 1, \quad \alpha \ll 1$.

Thanks to these assumptions, an analytical expression for $\Delta T_{k}$ can be obtained. Assuming $T_{0}=0$, we may state that the interval $\Delta T_{k}$ is in the proximity of $T=k \Delta T$. From Eqs. (14) and (15), the average driver velocity in the interval $\Delta T_{k}$ is then

$Y_{k} \simeq V(1+A \sin 2 \pi \alpha k)$ 

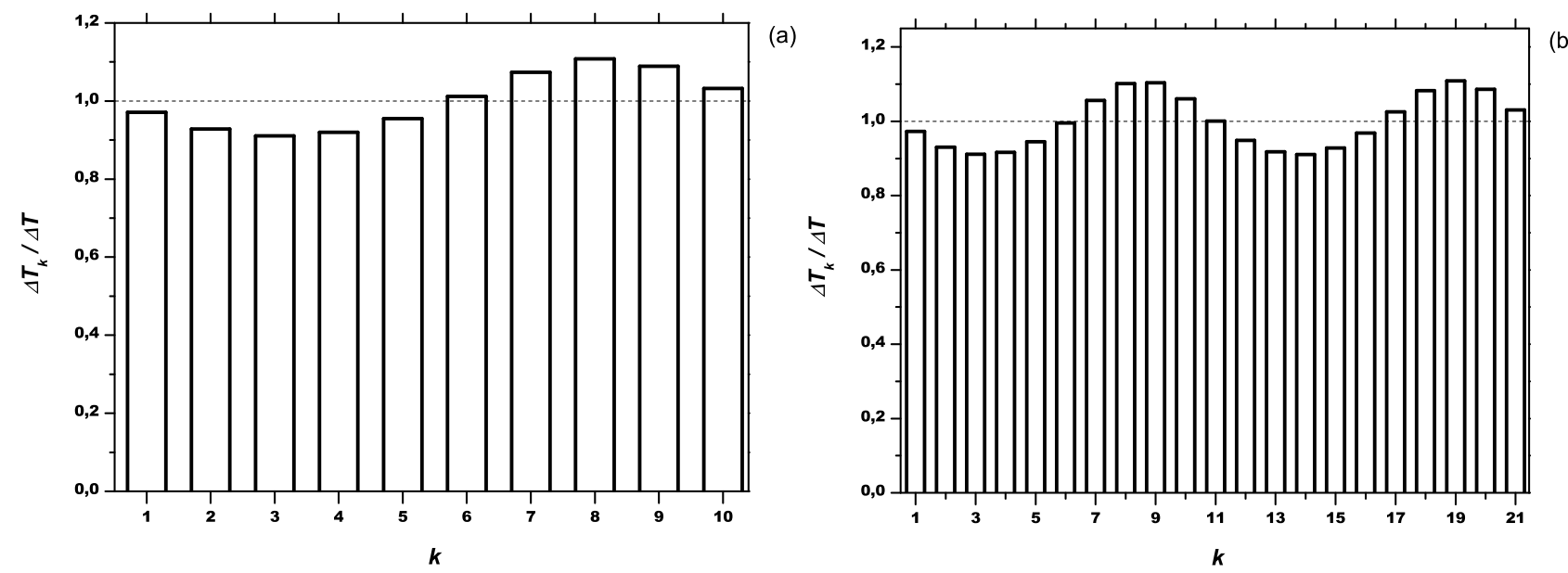

Fig. 3. Histograms showing the recurrence time $\Delta T_{k} / \Delta T$ as a function of $k$ : (a) $\alpha=1 / 10$, (b) $\alpha=2 / 21(\epsilon=0.7, A=0.1)$.

Then

$\Delta T_{k} \simeq \frac{U}{Y_{k}}$

or, thanks to Eq. (12) and with the same approximation,

$\Delta T_{k} \simeq \Delta T(1-A \sin 2 \pi \alpha k)$

showing that the values of $\Delta T_{k}$ as a function of $k$ belong to a sinusoid with wavelength $1 / \alpha$ oscillating about the value $\Delta T$ with amplitude $A$. Hence the nondimensional oscillation amplitude of recurrence times coincides with that of velocity oscillations, when $\alpha \ll 1$. From $\Delta T_{k}$ we can calculate $Z_{k}$ from Eqs. (25) and (33). Using Eq. (15), we obtain

$Z_{k}=2 \pi \alpha\left(k-A \sum_{i=1}^{k} \sin 2 \pi \alpha i\right)$

or, calculating the sum and setting $k+1 \simeq k$,

$Z_{k}=2 \pi \alpha\left(k-A \frac{\sin ^{2} \pi \alpha k}{\sin \pi \alpha}\right)$

showing that $Z_{k}$ is an increasing, nonlinear function of $k$. From Eqs. (25) and (15),

$T_{k}=\Delta T\left(k-A \frac{\sin ^{2} \pi \alpha k}{\sin \pi \alpha}\right)$.

With $\alpha$ given by Eq. (34), after a cycle including $n$ block motions Eq. (63) gives Eq. (36) and Eq. (64) gives Eq. (37). In the case of a monotonic velocity change, we may analogously assume

$|B| \ll 1, \quad \beta \ll 1$

which yields $T=k \overline{\Delta T}$ and
$Y_{k} \simeq V_{+}(1+B \cos 2 \pi \beta k)$

so that

$\Delta T_{k} \simeq \overline{\Delta T}(1-B \cos 2 \pi \beta k)$

describing a monotonic change of $\Delta T_{k}$ from $\Delta T_{a}$ to $\Delta T_{b}$. Then

$Z_{k}=2 \pi \beta\left(k-B \sum_{i=1}^{k} \cos 2 \pi \beta i\right)$

or, calculating the sum and setting $k \pm(1 / 2) \simeq k$,

$Z_{k}=2 \pi \beta\left(k-B \frac{\sin \pi \beta k \cos \pi \beta k}{\sin \pi \beta}\right)$

and finally

$T_{k}=\overline{\Delta T}\left(k-B \frac{\sin \pi \beta k \cos \pi \beta k}{\sin \pi \beta}\right)$.

\section{Discussion}

We considered a spring-block system with variable driver velocity as a model for the long-term behaviour of a fault subject to variable strain rate. In the case of sinusoidal oscillation, we may conclude that, while single earthquakes produced by the fault are aperiodic, the fault activity is characterized by seismic cycles made of a finite number of events. The number of events in a cycle depends on the ratio $\alpha$ between the frequency of strain rate oscillations and the frequency of earthquake occurrence at constant strain rate.

In order to illustrate the results, we choose $\epsilon=0.7$, a typical value for friction of rocks (e.g. Scholz, 1990). From Eq. (11) it follows $U=0.6$. We take initial conditions in 

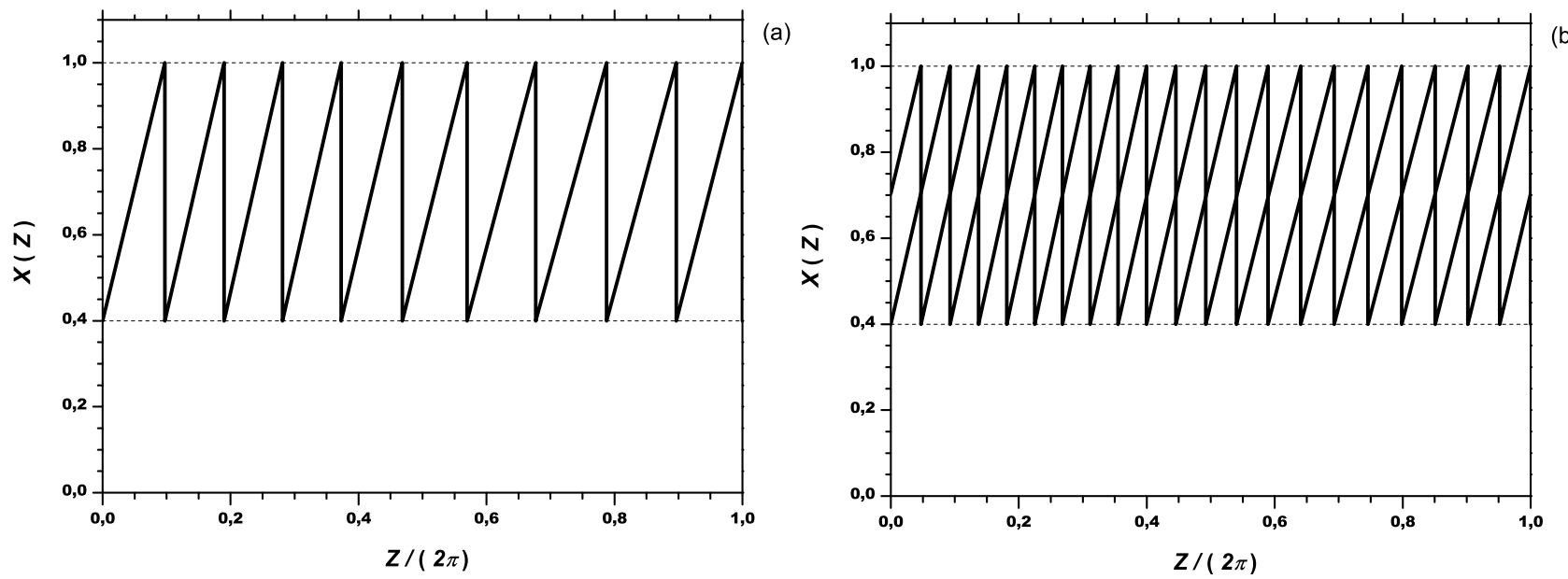

Fig. 4. Projections of the orbits in the plane $X Z$ : (a) $\alpha=1 / 10$, (b) $\alpha=2 / 21(\epsilon=0.7, A=0.1)$.
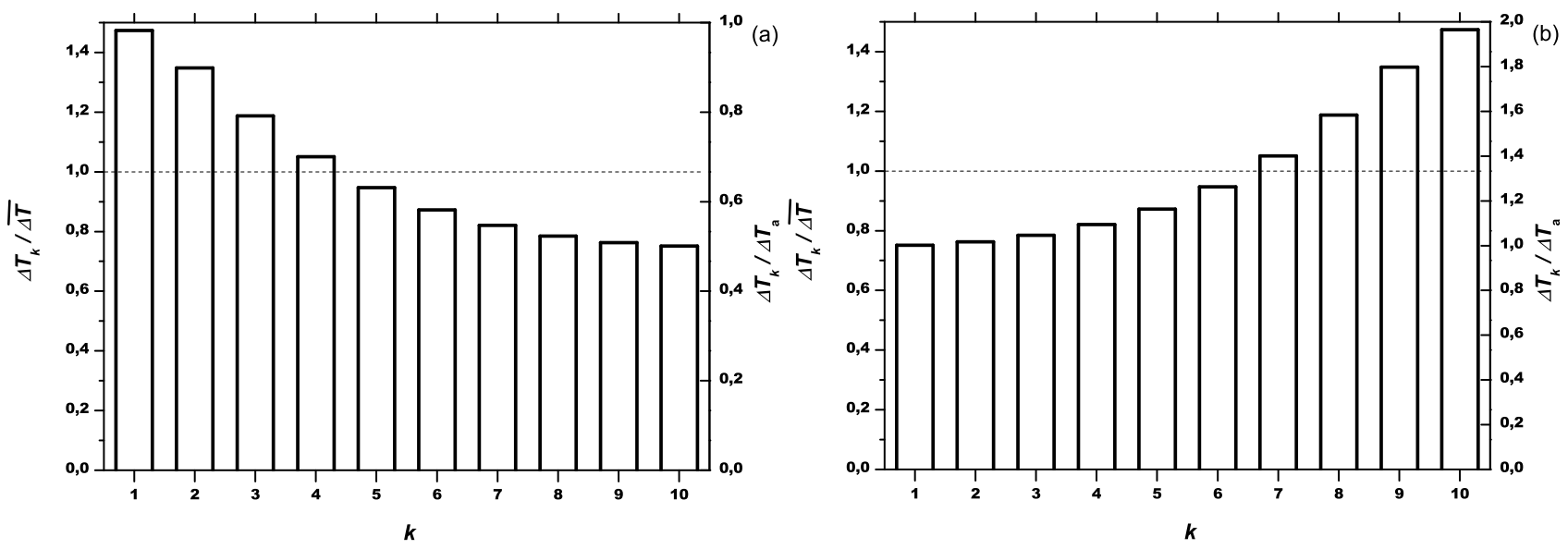

Fig. 5. Histograms showing the recurrence time $\Delta T_{k} / \overline{\Delta T}$ (or $\Delta T_{k} / \Delta T_{a}$ ) as a function of $k$ : (a) $V_{b} / V_{a}=2$, (b) $V_{b} / V_{a}=1 / 2(\beta=1 / 20$ ).

Eq. (23), where $X_{0}=0.4$. We choose $A=0.1$ for the amplitude of strain rate oscillations.

The histograms in Fig. 3 show the recurrence times $\Delta T_{k}$ calculated from Eq. (31), in the cases $\alpha=1 / 10$ and $\alpha=$ $2 / 21$. A complete seismic cycle is shown in both cases, with $\Delta T_{k}$ oscillating about $\Delta T$. The cycles include 10 and 21 earthquakes respectively and correspond to 1 and 2 cycles of strain rate oscillations, respectively.

Figure 4 shows the projections of the orbits in the plane $X Z$, calculated from Eq. (32) in the same two cases. In the case $\alpha=1 / 10$, there are 10 values of $Z_{k}$ in the interval $0<Z \leq 2 \pi$, with $Z_{10}=2 \pi$. Then exactly 10 earthquakes take place in the time interval $2 \pi / \Omega$. The tenth earthquake occurs at $T=T_{10}=10 \Delta T$. The system is in a periodic orbit with period $2 \pi / \Omega$, representing a sequence of 10 earthquakes with a certain pattern of recurrence times.
In the case $\alpha=2 / 21,2$ cycles of $Y$ are necessary in order that $Z_{k}$ is a multiple of $2 \pi$ and the initial conditions are recovered. There are 21 values of $Z_{k}$ in the interval $0<Z \leq 2 \pi$. Then 21 earthquakes take place in the time interval $4 \pi / \Omega$, with the twentieth earthquake occurring at $T=T_{21}=21 \Delta T$. The system in a periodic orbit with period $4 \pi / \Omega$. The seismic cycle is a sequence of 21 earthquakes, which are distributed over a time twice longer than in the former case. Therefore a small change in $\alpha$, about $5 \%$, produces a very different seismicity pattern.

In the case of a monotonic change in strain rate taking place in a finite time $\Theta$, the recurrence times change gradually from the value $\Delta T_{a}$ preceding the change to the value $\Delta T_{b}$ following it. In order to illustrate this case, we choose $\beta=1 / 20$, so that exactly 10 earthquakes occur in the time interval $\Theta$. The histograms in Fig. 5 show the recurrence times 
$\Delta T_{k}$ calculated from Eq. (51), in the cases $V_{b} / V_{a}=2$ and $V_{b} / V_{a}=1 / 2$. They are also expressed in terms of $T_{a}$ thanks to the equation

$\Delta T_{a}=\frac{1}{2}\left(1+\frac{V_{b}}{V_{a}}\right) \overline{\Delta T}$

In general, the seismic cycles associated with sinusoidal oscillations and the transition intervals between two strain rate values may include a large number of events and hence may be very long and difficult to observe even if the fault were subject only to tectonic strain rate. However most faults are part of a fault system and are subject to stress transfers from neighbouring faults in connection with earthquakes. If we suppose that an amount of tangential stress is transferred to the fault during the time interval $\Delta T_{k}$, this results in an abrupt change $\Delta X$ superimposed to the saw-tooth curve Eq. (32). The orbit is then shifted to

$X_{k}^{\prime}(Z)=1+U \frac{Z-Z_{k}}{Z_{k}-Z_{k-1}}+\Delta X$

which reaches the value $X=1$ when $Z$ is equal to

$Z_{k}^{\prime}=Z_{k}-\frac{\Delta X}{U}\left(Z_{k}-Z_{k-1}\right)$.

If we subtract $Z_{k-1}$ from both sides and multiply by $\Delta T /(2 \pi \alpha)$ or $\overline{\Delta T} /(2 \pi \beta)$, we obtain

$\Delta T_{k}^{\prime}=\left(1-\frac{\Delta X}{U}\right) \Delta T_{k}$

Hence the $k$-th earthquake is anticipated or delayed by an amount $\Delta X / U$, independent of $\alpha$ or $\beta$. If $\Delta X>0$, the earthquake is anticipated; if $\Delta X<0$, it is delayed. According to Eq. (8), $\Delta X$ expresses the stress change as a fraction of static friction. For example, if $\Delta X=3 \%$ the relative change in $\Delta T_{k}$ is equal to $5 \%$, with the choice made for $\epsilon$.

The stress transfer modifies the following recurrence times. In the case of sinusoidal oscillations, the current seismic cycle stops and a new one starts with different initial conditions, but with the same period $2 \pi \mathrm{m} / \Omega$. If the fault system is made of several faults, the frequency of stress perturbations may be higher than $\Omega$, thus preventing the fault from completing any regular seismic cycle. In the case of a monotonic change, a stress transfer from neighbouring faults will analogously modify the evolution of recurrence times. An accumulation of stress perturbations with the same sign can change the number of seismic events taking place during the transition.

\section{Conclusions}

A seismogenic fault is a complicated system, characterized by a heterogeneous friction and subject to various influences, such as tectonic strain rate and stress transfers from neighbouring faults. We have greatly simplified this picture considering a single, isolated fault and describing its mechanics with an equivalent low-order system. The aim is to understand the effect of a slowly variable strain rate on the recurrence times of earthquakes.

In the case of constant strain rate, the fault produces earthquakes with a recurrence period $\Delta T$. Variable strain rate is a cause of aperiodicity, if we look at single earthquakes. In the case of a sinusoidally varying strain rate, the seismic activity of the fault organizes into cycles that include several earthquakes and repeat periodically. The period of such seismic cycles is a multiple of the oscillation period of strain rate. Within each cycle the recurrence times oscillate about the average value $\Delta T$ and the amplitude of oscillations is proportional to that of strain rate oscillations. In the case of a monotonic change in strain rate, the recurrence times change gradually from the value preceding the change to the one following it.

If the fault is subject to perturbations in stress, the perturbation anticipates or delays the subsequent earthquake. In the case of sinusoidal oscillations, each perturbation will break off the current seismic cycle and will start a new one. Therefore the pattern of seismic cycles controlled by strain rate oscillations may be spoiled in the presence of frequent stress transfers, as may occur in systems made of several interacting faults. In the case of a monotonic change, stress perturbations will similarly alter the regular pattern of recurrence times and several perturbations with the same sign can change the number of earthquakes occurring during the transition.

In summary, even if slow variations in strain rate may be difficult to observe in seismicity records due to the presence of external perturbations, it is undoubted that such variations give a significant contribution to the duration of recurrence times and to the observed aperiodicity of earthquakes.

Acknowledgements. The authors are grateful to Leigh Phoenix, to an anonymous referee and to the editor William I. Newman for constructive comments on the first version of the paper.

Edited by: W. I. Newman

Reviewed by: L. Phoenix and another anonymous referee

\section{References}

Abaimov, S. G., Turcotte, D. L., Shcherbakov, R., and Rundle, J. B.: Recurrence and interoccurrence behavior of self-organized complex phenomena, Nonlin. Processes Geophys., 14, 455-464, doi:10.5194/npg-14-455-2007, 2007.

Baiesi, M.: Correlated earthquakes in a self-organized model, Nonlin. Processes Geophys., 16, 233-240, doi:10.5194/npg-16-2332009, 2009.

Belardinelli, M. E. and Belardinelli, E.: The quasi-static approximation of the spring-slider motion, Nonlin. Processes Geophys., 3, 143-149, doi:10.5194/npg-3-143-1996, 1996.

Ben-Zion, Y., Eneva, M., and Liu, Y.: Large earthquake cycles and intermittent criticality on heterogeneous faults due to evolv- 
ing stress and seismicity, J. Geophys. Res., 108(B6), 2307, doi:10.1029/2002JB002121, 2003.

Burridge, R. and Knopoff, L.: Model and theoretical seismology, Bull. Seismol. Soc. Am., 57, 341-371, 1967.

Byerlee, J.: Friction of rocks, Pure Appl. Geophys., 116, 616-626, 1978.

Cao, T. and Aki, K.: Seismicity simulation with a mass-spring model and a displacement hardening-softening friction law, Pure Appl. Geophys., 122, 10-23, 1984.

Cao, T. and Aki, K.: Seismicity simulation with a rate and state dependent friction law, Pure Appl. Geophys., 124, 487-513, 1986.

Carlson, J. M. and Langer, J. S.: Mechanical model of an earthquake fault, Phys. Rev. A, 40(11), 6470-6484, 1989a.

Carlson, J. M. and Langer, J. S.: Properties of earthquakes generated by fault dynamics, Phys. Rev. Lett., 62(22), 2632-2635, $1989 b$.

Carlson, J. M., Langer, J. S., and Shaw, B.: Dynamics of earthquake fault, Rev. Mod. Phys., 66(2), 657-659, 1994.

Cohen, S.: Computer simulation of earthquakes, J. Geophys. Res., 82, 3781-3796, 1977.

de Sousa Vieira, M.: Chaos in a simple spring-block system, Phys. Lett. A, 198, 407-414, 1995.

Dragoni, M. and Santini, S.: Simulation of the long-term behaviour of a fault with two asperities, Nonlin. Processes Geophys., 17, 777-784, doi:10.5194/npg-17-777-2010, 2010.

Dieterich, J. H.: Time dependent friction as a possible mechanism for aftershocks, J. Geophys. Res., 77, 3771-3781, 1972.

Erickson, B., Birnir, B., and Lavalle, D.: A model for aperiodicity in earthquakes, Nonlin. Processes Geophys., 15, 1-12, doi:10.5194/npg-15-1-2008, 2008.

Gu, J. C., Rice J. R., Ruina, A. L., and Tse S. T.: Slip motion and stability of a single degree of freedom elastic system with rate and state dependent friction, J. Mech. Phys. Sol., 32, 167-196, 1984.

Gu, J. C. and Wong T. F.: Effects of loading velocity, stiffness and inertia on the dynamics of a single degree of freedom springslider system, J. Geophys. Res., 96, 21677-21691, 1991.

$\mathrm{He}, \mathrm{C} .:$ Interaction between two sliders in a system with rate- and state-dependent friction, Science in China, Series D, 46, 67-74, 2003.

Huang, J. and Turcotte, D. L.: Are earthquakes an example of deterministic chaos?, Geophys. Res. Lett., 17, 223-226, 1990a.

Huang, J. and Turcotte, D. L.: Evidence for chaotic fault interactions in the seismicity of the San Andreas fault and Nankai trough, Nature, 348, 234-236, 1990b.

Huang, J. and Turcotte, D. L.: Chaotic seismic faulting with massspring model and velocity-weakening friction, Pure Appl. Geophys., 138, 569-589, 1992.
Iaffaldano, G. and Bunge, H.-P.: Relating rapid plate-motion variations to plate-boundary forces in global coupled models of the mantle/lithosphere system: Effects of topography and friction, Tectonophysics, 474, 393-404, 2009.

King, S. D., Lowman, J. P., and Gable, C. W.: Episodic tectonic plate reorganizations driven by mantle convection, Earth Planet. Sci. Lett., 203, 83-91, 2002.

Marzocchi, W., Selva, J., Piersanti, A., and Boschi, E.: On the long term interaction among earthquakes: Some insight from a model simulation, J. Geophys. Res., 108, 2538, doi:10.1029/2003JB002390, 2003.

Matthews, M. V., Ellsworth, W. L., and Reasenberg, P. A.: A Brownian model for recurrent earthquakes, Bull. Seism. Soc. Am., 92, 2233-2250, 2002.

McCloskey, J. and Bean, C. J.: Time and magnitude predictions in shocks due to chaotic fault interactions, Geophys. Res. Lett., 19, 119-122, 1992.

Nussbaum, J. and Ruina, A.: A two degree-of-freedom earthquake model with static/dynamic friction, Pure Appl. Geophys., 125, 629-656, 1987.

Reid, H. F.: The elastic-rebound theory of earthquakes, University of California Publ. Geol. Sci., 6, 413-444, 1911.

Rice, J. R. and Tse, S. T.: Dynamic motion of a single degree of freedom system following a rate and state dependent friction law, J. Geophys. Res., 91, 521-530, 1986.

Ruina, A.: Slip instability and state variable friction laws, J. Geophys. Res., 88, 10359-10370, 1983.

Rundle, J. B. and Jackson, D. D.: Numerical simulation of earthquake sequences, Bull. Seismol. Soc. Am., 67, 1363-1377, 1977.

Scholz, C. H.: The Mechanics of Earthquakes and Faulting, Cambridge University Press, Cambridge, 1990.

Steacy, S., Gomberg, J. and Cocco, M.: Introduction to special section: Stress transfer, earthquake triggering, and timedependent seismic hazard, J. Geophys. Res., 110, B05S01, doi:10.1029/2005JB003692, 2005.

Turcotte, D. L.: Fractals and Chaos in Geology and Geophysics, 2nd Edn., Cambridge University Press, Cambridge, 1997.

Zöller, G. and Hainzl, S.: Recurrence time distributions of large earthquakes in a stochastic model for coupled fault systems: The role of fault interaction, Bull. Seism. Soc. Am., 97, 1679-1687, 2007.

Zöller, G., Ben-Zion, Y., Holschneider, M., and Hainzl, S.: Estimating recurrence times and seismic hazard of large earthquakes on an individual fault, Geophys. J. Int. 170, 1300-1310, doi:10.1111/j.1365-246X.2007.03480.x, 2007. 